\title{
ORGANIZAÇÃO SOCIAL E INDICADORES SOCIOECONÔMICOS NO BRASIL: um estudo exploratório
}

\author{
Sylvio Bandeira de Mello e Silva* \\ Barbara-Christine Nentwig Silva* \\ Maina Pirajá Silva***
}

\begin{abstract}
O trabalho tem como objetivo analisar a distribuição espacial das organizações sociais no Brasil e correlacionar esses resultados com dados socioeconômicos. Como indicadores das organizações sociais que exercem funções na sociedade, foram tomadas as informações do IBGE sobre as fundações privadas e associações sem fins lucrativos. Já os indicadores socioeconômicos são os seguintes: Indice dos desequilíbrios regionais de Williamson, PIB per capita, Indice de desenvolvimento humano (IDH), Índice de Gini, Taxa de mortalidade infantil, Esperança de vida ao nascer e Média de anos de estudo (20 a 24 anos). Os resultados, após o emprego de uma metodologia de análise estatística e cartográfica em diferentes escalas do território nacional, indicam uma variedade de situações, mas é possível afirmar que, para os estados com melhor relação entre habitantes por organizações sociais, existe uma correlação significativa com melhores indicadores econômico-sociais. Assim, há relevância dos resultados para o entendimento da diversidade geográfica brasileira.
\end{abstract}

PALAVRAS-CHAVE: organização social, capital social, indicadores socioeconômicos, desenvolvimento, Brasil.

\section{INTRODUÇÃO}

O trabalho tem como objetivo realizar um mapeamento da diversidade da distribuição das organizações sociais no Brasil, por grandes regiões e por estados, comparando esses dados com outros sobre desequilíbrios regionais e desigualdades sociais.

Com isso, busca-se identificar tipos de correspondência entre os indicadores do potencial de organização social em nosso contexto e os referentes aos desequilíbrios econômicos e desigualdades sociais. A questão básica que se coloca é se haveria uma relação entre padrões mais elevados de organização social com desequilíbrios econômicos menos expressivos e com bons indicadores sociais.

* Professor do Programa de Pós-graduação em Planejamento Territorial e Desenvolvimento Social/UCSAL e do Mestrado em Geografia/UFBA. Pesquisador/CNPq Grupo de Pesquisa GLOCAL.

Av. Cardeal da Silva, 205. Cep: 40.231-902. Federação Salvador - Bahia - Brasil. sylvioms@ucsal.br

* * Professora do Programa de Pós-graduação em Planejamento Territorial e Desenvolvimento Social/UCSAL e do Mestrado em Geografia/UFBA. Pesquisadora/CNPq Grupo de Pesquisa GLOCAL. barbarans@ucsal.br

*** Licenciada em Geografia/UFBA. Grupo de Pesquisa GLOCAL/CNPq. mainapiraja@yahoo.com.br

\section{ASPECTOS CONCEITUAIS, MATERIAIS E MÉTODOS}

Na análise da dinâmica da organização social, destaca-se um conjunto de ideias em torno do que se convencionou chamar de capital social, sobretudo a partir das contribuições pioneiras de Bourdieu ([1980] 1998), Coleman (1988, 1990) e Putnam ([1993] 1996). Bourdieu segue mais uma abordagem estruturalista nas relações entre os agentes, Coleman, a do institucionalismo (papel das instituições e do individualismo, um recurso para as pessoas) e Putnam, a do institucionalismo. Apesar dessas diferenças, os autores citados ressaltam a importância das organizações sociais para a introdução e direcionamento de atividades inovadoras no sistema econômico-social.

Assim, por exemplo, Putnam (1996, p.177), mais utilizado neste trabalho em função de sua maior aderência à metodologia, define capital social como o conjunto de "características da organização social, como confiança, normas e sistemas que contribuem para aumentar a eficiência da sociedade, facilitando as ações coordenadas". Em seu de- 
talhado estudo sobre a Itália, o de maior referência neste campo, esse conceito foi básico para entender a diversidade de situações relacionadas com os diferentes níveis de desenvolvimento existentes na península italiana.

Em um extenso trabalho de avaliação teórica e aplicada sobre capital social e desenvolvimento econômico, Woolcock (1998) afirma que:

[...] development outcomes are shaped by the extent to which basic social dilemmas at the micro and macro level are resolved. Positive outcomes are attained to the extent that both embedded and autonomous social relations prevail at both levels. This happens when people are willing and able to draw on nurturing social ties (i) within their local communities; (ii) between local communities and groups with external and more extensive social connection to civil society; (iii) between civil society and macro-level institutions; and (iv) within corporate sector institutions. All four dimensions must be present for optimal development outcomes. (Woolcock, 1998, p.186) ${ }^{1}$

\section{E, finalmente, afirma que o capital social}

[...] make potentially important contributions to questions of economic development, contributions that complement orthodox economic approaches in some respects and challenge them in others. Social capital's greatest merit, however, is that it provides a credible point of entry for sociopolitical issues into a comprehensive multi- and interdisciplinary approach to some of the most pressing issues of our time. (Woolcock, 1998, p.188) ${ }^{2}$

Já outro pesquisador (Becker, 2003) questiona, inicialmente, “a dimensão inovadora contida na concepção ideológica de capital social” (p. 88) e resgata, a seguir, contribuições pretéritas, como as de bloco social regional e grupo territorial hegemônico,

... as perspectivas do desenvolvimento são moldadas pela extensão da solução dos dilemas sociais em um níve micro e macro. Resultados positivos são atingidos através da extensão da prevalência das relações sociais enraizadas e autônomas nos dois níveis. Isso acontece quando a pessoas desejam e são capazes de desenhar e nutrir relações sociais (i) em suas comunidades; (ii) entre comunidades locais e grupos com relações externas e conexões sociais mais extensivas para a sociedade civil; (iii) entre a sociedade civil e as instituições em um nível macro; e (iv) no contexto das instituições do setor empresarial. Todas as quatro dimensões devem estar presentes para a emergência do desenvolvimento ótimo.

2 ...traz potencialmente importantes contribuições para questões do desenvolvimento econômico, contribuiçõe que complementam abordagens econômicas ortodoxas em alguns aspectos e as desafia em outros. O maio mérito do capital social, entretanto, é que ele oferece um crédito para colocar as questões sócio-políticas em uma abordagem compreensiva multi e interdisciplinar como as mais prementes questóes de nosso tempo. de Gramsci (p. 111), e a de empresários inovadores, de Schumpeter (p. 112). Isso permite ao autor concluir com a tese de que

uma sociedade mais organizada socialmente é uma sociedade mais participativa politicamente. E uma sociedade mais participativa politicamente é uma sociedade muito mais desenvolvida economicamente (Becker, 2003, p.116).

Evidentemente, pensadores marxistas ou de outras correntes críticas, não discutem a perspectiva do capital social, preferindo contribuir com análises de questões estruturais expressas, por exemplo, pelos movimentos sociais relacionados com a luta de classes e com o papel do Estado em uma sociedade capitalista. Quando citam Putnam, só o fazem, sobretudo, na bibliografia, mas não discutem suas ideias no corpo do livro, como o fez Harvey (2000, 2001), importante geógrafo marxista da atualidade. Entretanto, seu conceito de "governança urbana”, incorporando “o papel facilitador e coordenador do governo e da administração urbana" ao "conjunto complexo de forças mobilizado por diversos agentes sociais”, passando pela "iniciativa cívica”, se aproxima do conceito de capital social (Harvey [1989] 2005, p.171). Putnam, que tanto valoriza a participação cívica, por sua vez, não cita Marx e Harvey em sua obra. Porém se aproxima de Harvey quando diz: "O conceito de desempenho institucional baseia-se num modelo bem simples de governança: demandas sociais $\rightarrow$ interação política $\rightarrow$ governo $\rightarrow$ opção de política $\rightarrow$ implementação.” (Putnam, 1996, p.24). Há, portanto, importantes aproximações entre diferentes correntes do pensamento que destacam a perspectiva da dinâmica da organização social em sua relação com o desenvolvimento econômico e social.

Em nosso estudo sobre o Brasil, tomamos como indicador da capacidade organizacional da sociedade brasileira, ou seja, do seu capital social, a distribuição das Fundações Privadas e Associações sem Fins Lucrativos (FASFIL), tal como fornecida pelo Instituto Brasileiro de Geografia e Estatística (IBGE), em parceria com a Associação Brasileira de Organizações não Governamentais (ABONG), o Grupo de Institutos Fundações e 
Empresas (GIFE) e o Instituto de Pesquisa Econômica Aplicada (IPEA). Putnam (1996), em sua obra, valoriza bastante a análise das organizações sociais, ao lado de outros aspectos como confiança em instituições, participação cívica e solidariedade.

A metodologia para a definição das FASFIL foi a de selecionar, no Cadastro Central de Empresas (CEMPRE/IBGE), para 2005, as entidades, com base na composição de sua natureza jurídica que se enquadrassem, "simultaneamente, nos cinco seguintes critérios:

- privadas, não integrantes, portanto, do aparelho de Estado;

- sem fins lucrativos, isto é, organizações que não distribuem eventuais excedentes entre os proprietários ou diretores e que não possuem como razão primeira de existência a geração de lucros - podendo até gerá-los, desde que aplicados nas atividades fins;

- institucionalizadas, istoé, legalmente constituídas; - autoadministradas ou capazes de gerenciar suas próprias atividades; e

- voluntárias, na medida em que podem ser constituídas livremente por qualquer grupo de pessoas, isto é, a atividade de associação ou de fundação da entidade é livremente decidida pelos sócios ou fundadores” . (IBGE, 2008, p.12-13)

Os indicadores econômico-sociais selecionados são os seguintes: PIB per capita (IBGE), Índice de Williamson (calculado pelos autores com base em dados do IBGE), Índice de Gini (IPEA), Índice de desenvolvimento humano (PNUD), Taxa de mortalidade infantil (IBGE), Esperança de vida ao nascer (IBGE) e Média de anos de estudo - 20 a 24 anos (IBGE). As informações foram processadas combinando-se métodos de análise estatística e cartográfica.
Gráfico 1 - Brasil - Fundações privadas e associações sem fins lucrativos por setores de atividade $-\mathbf{2 0 0 5}$

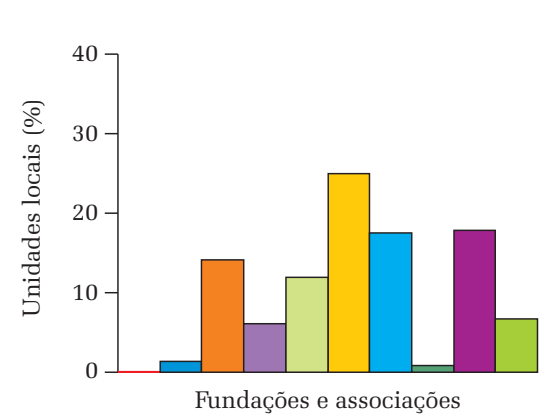

Fonte dos dados: IBGE, 2008, p. 27 Elaboração: própria.

\section{ANÁLISE DAS INFORMAÇÕES SOBRE FUN- DAÇÕES E ASSOCIAÇÕES SEM FINS LUCRA- TIVOS}

Inicialmente, a distribuição das FASFIL em 2005, em termos relativos e por setores de atividade, pode ser vista no Gráfico 1, onde aparecem como os mais importantes os seguintes setores: Religião, Desenvolvimento e Defesa de Direitos e Associações Patronais e Profissionais.

Surpreendentemente, os setores de Habitação, de Saúde e de meio ambiente e proteção animal apresentam, no total, um pequeno número. $\mathrm{O}$ setor de habitação representa só $0,1 \%$ e, por isso, não se destaca no Gráfico 1 . Nos dois primeiros casos, provavelmente a causa está relacionada às dificuldades de implantação de FASFIL em setores que normalmente detém elevada participação do Estado e de empresas privadas. Já no caso do meio ambiente e proteção animal, é preciso destacar, além da presença do Estado, a participação de organizações não-institucionalizadas, especialmente para o meio ambiente. Entretanto, entre 1970 e 2005, o setor de meio ambiente e proteção animal é o segundo setor que mais cresceu, como mostra a Tabela 1.É justamente nesse período que as questões ambientais adquirem maior relevância em todo o mundo.

A distribuição absoluta das FASFIL por estados, relativizada pela população de cada unidade, é apresentada na Tabela 2. Observa-se que os três estados do Sul estão, em termos relativos, em 
Tabela 1 - Brasil - Número de fundações privadas e associações sem fins lucrativos - 1970/2005

\begin{tabular}{l|r|r|r}
\hline Classificação das entidades sem fins lucrativos & 1970 & 2005 & Proporção de crescimento \\
\hline Habitação & 6 & 456 & 76,00 vezes \\
Saúde & 921 & 4.464 & 4,85 vezes \\
Cultura e recreação & 2.050 & 46.999 & 22,93 vezes \\
Educação e pesquisa & 1.435 & 19.940 & 13,90 vezes \\
Assistência social & 1.966 & 39.395 & 20,04 vezes \\
Religião & 3.082 & 83.775 & 27,18 vezes \\
Associações patronais e profissionais & 702 & 58.796 & 83,75 vezes \\
Meio ambiente e proteção animal & 16 & 2.562 & 160,13 vezes \\
Desenvolvimento e defesa de direitos & 276 & 60.259 & 218,33 vezes \\
Outras fundações provadas e associações sem fins lucrativos & 485 & 21.516 & 44,36 vezes \\
Total & 10.939 & 338.162 & 30,91 vezes \\
\hline
\end{tabular}

Fonte dos dados: IBGE, 2008, p. 31.

Elaboração: própria.

Tabela 2 - Brasil e Unidades da Federação

Pessoas por fundações privadas e associações sem fins lucrativos - 2005

\begin{tabular}{|c|c|c|c|}
\hline $\begin{array}{l}\text { Unidades } \\
\text { da Federação }\end{array}$ & $\begin{array}{c}\text { Pessoas por } \\
\text { fundações e associações }\end{array}$ & $\begin{array}{l}\text { Unidades da } \\
\text { Federação }\end{array}$ & $\begin{array}{c}\text { Pessoas por } \\
\text { fundações e associações }\end{array}$ \\
\hline Santa Catarina & 269 & São Paulo & 589 \\
\hline Rio Grande do Sul & 366 & Bahia & 595 \\
\hline Paraná & 403 & Acre & 598 \\
\hline Piauí & 453 & Rio Grande do Norte & 618 \\
\hline Minas Gerais & 466 & Sergipe & 639 \\
\hline Espírito Santo & 496 & Tocantins & 698 \\
\hline Distrito Federal & 513 & Maranhão & 707 \\
\hline Mato Grosso do Sul & 530 & Goiás & 727 \\
\hline Paraíba & 545 & Roraima & 758 \\
\hline Brasil & 545 & Pernambuco & 836 \\
\hline Mato Grosso & 548 & Pará & 1.055 \\
\hline Ceará & 555 & Amazonas & 1.112 \\
\hline Rondônia & 558 & Alagoas & 1.286 \\
\hline Rio de Janeiro & 577 & Amapá & 1.532 \\
\hline
\end{tabular}

Fonte dos dados: IBGE, 2008, p. 24.

Elaboração: própria.

melhor situação, ou seja, é preciso reunir um menor número de pessoas para se encontrar uma fundação ou associação. A presença do Piauí em $4^{\circ}$ lugar é uma questão instigante que merece ser pesquisada em outro trabalho.

A distribuição espacial fica ressaltada melhor no Mapa 1, onde é bem evidente o contraste da região Sul com a região Norte. Em termos gerais, esse mapa se aproxima bastante da realidade dos desequilíbrios econômicos e desigualdades sociais atualmente vigentes no Brasil (Diniz, 1993, 2000; Santos; Silveira, 2001; Silva; Silva; Coelho, 2008).

Já a Tabela 3 apresenta a variação dos salários pagos pelas FASFIL, para o Brasil e para as grandes regiões, onde se destaca a região Sudeste com os maiores valores, contrastando com o Nordeste com os menores salários médios mensais. 
Sylvio Bandeira de Mello e Silva, Barbara-Christine

Nentwig Silva, Maina Pirajá Silva

Mapa 1 - Brasil - Unidades da Federação - Pessoas por fundações privadas e associações sem fins lucrativos - 2005

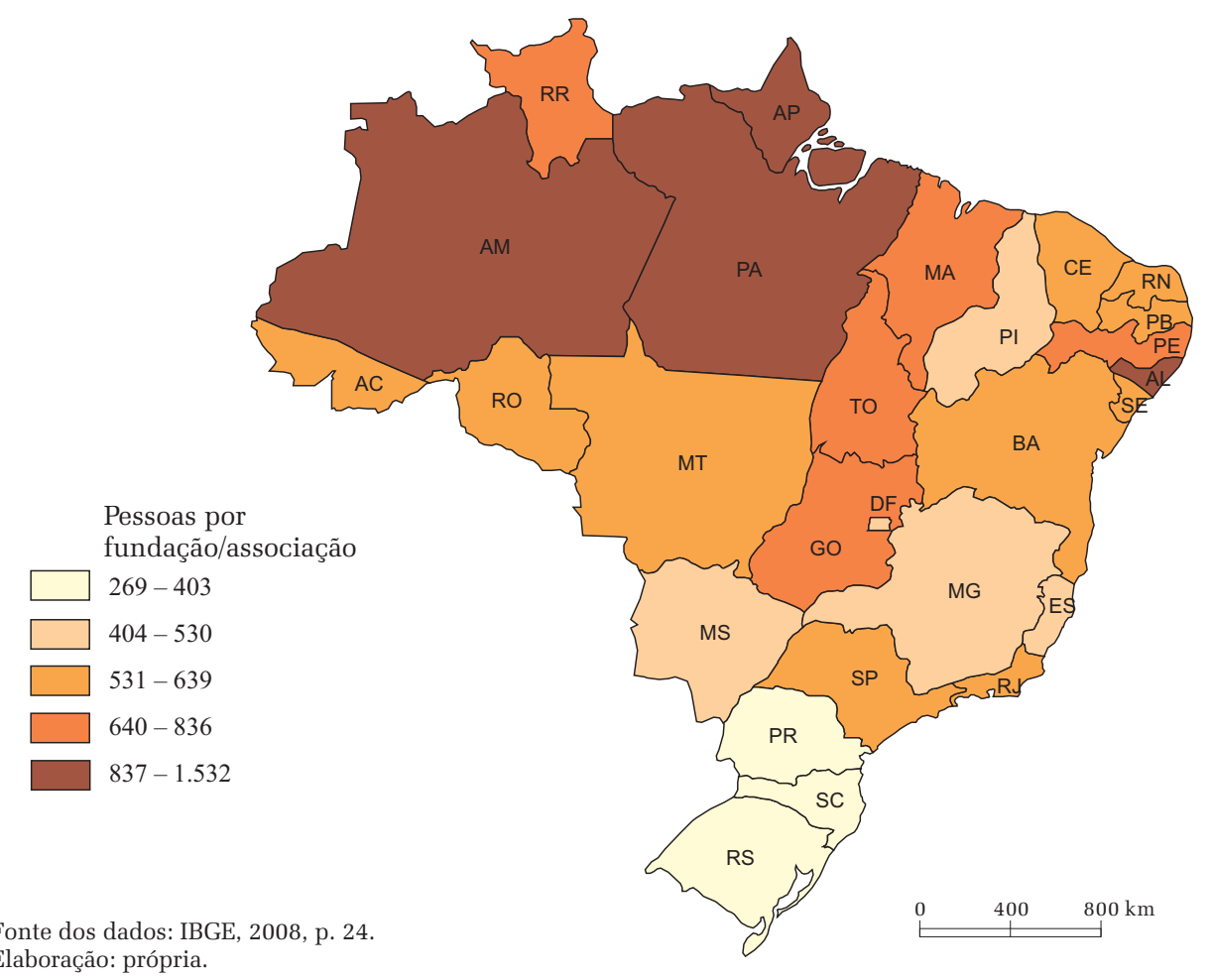

Elaboração: própria.

Tabela 3 - Brasil - Regiões - Salários médios mensais* pagos pelas fundações privadas e associações sem fins lucrativos -2005

\begin{tabular}{l|c|c|c|c|c|c}
\hline $\begin{array}{l}\text { Classificação das entidades } \\
\text { sem fins lucrativos }\end{array}$ & Brasil & Norte & Nordeste & Sudeste & Sul & $\begin{array}{l}\text { Centro- } \\
\text { Oeste }\end{array}$ \\
\hline Total & 3,8 & 3,1 & 2,7 & 4,1 & 3,9 & 3,4 \\
Habitação & 2,8 & - & 2,5 & 2,9 & 1,5 & 5,3 \\
Saúde & 3,6 & 2,8 & 2,9 & 3,8 & 3,4 & 3,0 \\
Cultura e recreação & 3,8 & 3,0 & 1,8 & 4,0 & 4,0 & 3,5 \\
Educação e pesquisa & 5,1 & 3,9 & 3,6 & 5,6 & 5,1 & 4,6 \\
Assistência social & 2,6 & 2,6 & 2,0 & 2,7 & 2,5 & 2,7 \\
Religião & 2,5 & 2,0 & 1,9 & 2,8 & 2,5 & 2,2 \\
Associações patronais & 3,3 & 3,0 & 1,5 & 3,7 & 3,9 & 3,0 \\
e profissionais & 4,3 & 3,8 & 4,9 & 4,4 & 3,2 & 4,2 \\
$\begin{array}{l}\text { Meio ambiente e } \\
\text { proteção animal }\end{array}$ & 3,5 & 3,0 & 2,1 & 3,5 & 4,9 & 2,7 \\
$\begin{array}{l}\text { Desenvolvimento } \\
\text { e defesa de direitos }\end{array}$ & 3,8 & 3,0 & 3,0 & 4,5 & 3,9 & 2,5 \\
$\begin{array}{l}\text { Outras fundações } \\
\text { privadas e associações } \\
\text { sem fins lucrativos }\end{array}$ & & & & & \\
\hline
\end{tabular}

* Em salário mínimo de 2005 (R\$ 286,67).

Fonte dos dados: IBGE, 2008, p. 41.

Elaboração: própria. 
ORGANIZAÇÃO SOCIALE DESENVOLVIMENTO: comparações e reflexões

Após a análise dos dados da distribuição das FASFIL, tomados como indicadores do capital social, é importante relacionar essas informações com outras, na perspectiva de buscar uma integração sobre a complexidade dos níveis de desenvolvimento no Brasil.

A primeira relação, apresentada de forma gráfica, é entre o total de Pessoas por fundações e associações com o PIB per capita por grandes regiões brasileiras (Tabela 4). posição. Dois casos extremos chamam a atenção: o já mencionado Piauí, com bons indicadores de Pessoas por fundações e associações e o menor PIB per capita e São Paulo, com indicadores médios de Pessoas por FASFIL e a segunda mais alta renda per capita, após o Distrito Federal que desfruta de uma situação particular por ser a capital do país.

A comparação é também instigante entre Pessoas por fundações e associações e o Índice de desequilíbrios regionais intraestaduais, com base em microrregiões, e de acordo com a metodologia proposta por Williamson ([1965], 1977). O Índice
Tabela 4 - Brasil - Regiões - Relação entre Pessoas por fundações privadas e associações sem fins lucrativos e PIB per capita - 2005

\begin{tabular}{|c|c|c|c|}
\hline Regiões & $\begin{array}{c}\text { Pessoas } \\
\text { por fundações } \\
\text { e associações }\end{array}$ & Regiões & $\begin{array}{c}\text { PIB } \\
\text { per capita }\end{array}$ \\
\hline Sul & 351 & Sudeste & 15.468 \\
\hline Sudeste & 547 & Centro-Oeste & 14.604 \\
\hline Centro-Oeste & $601-$ & Sul & 13.208 \\
\hline Nordeste & $638-$ & Norte & 7.247 \\
\hline Norte & $909-$ & Nordeste & 5.498 \\
\hline
\end{tabular}

Fonte dos dados: IBGE, 2008; IBGE, 2002-2005. Elaboração: própria.

Observa-se que há uma correspondência parcial entre os dois indicadores. O Sul cai do $1^{\circ}$ lugar em Pessoas por FASFIL para o $3^{\circ}$ lugar em PIB per capita; o Sudeste sobe do $2^{\circ}$ para o $1^{\circ} \mathrm{lu}-$ gar, o Centro-Oeste do $3^{\circ}$ para o $2^{\circ}$ lugar, o Nordeste cai do $4^{\circ}$ para o $5^{\circ}$ e último lugar, e o Norte sobe do $5^{\circ}$ para o $4^{\circ}$ lugar. O PIB do Centro-Oeste é certamente influenciado pelo PIB do Distrito Federal, como veremos a seguir.

Essa mesma comparação é feita para as Pessoas por fundações e associações e PIB per capita para as unidades da Federação (Tabela 5) onde, das 27 unidades, 11 apresentam uma relação relativamente significativa, ou seja, com mudança da posição hierárquica de cada estado em até três posições, considerando a ordem hierárquica das duas variáveis. Assim, por exemplo, Santa Catarina muda do $1^{\circ}$ lugar para o $4^{\circ}$ lugar, comparando-se as duas variáveis. Já Alagoas muda somente uma
deWilliamson combina a população de um determinado país ou estado, a população das regiões, o PIB per capita nacional ou estadual e o PIB per capita das regiões. Quanto mais próximo de zero, menor será o desequilíbrio regional de um de-

terminado país ou estado. A relação aqui (Tabela 6) é menos significativa do que no exemplo anterior (Tabela 5). Somente sete estados mudam de posição hierárquica em até três posições. Há casos igualmente extremos como o do Amapá e o do Piauí, por exemplo. Amapá é certamente um estado mais homogêneo em seus indicadores, considerando sua base econômica e sua estrutura urbano-regional pouco complexa. Já o Piauí tem uma base econômica e uma estrutura urbano-regional bem mais heterogênea que o estado do Amapá, o que resulta em maiores índices de desequilíbrios regionais.

Por outro lado, a Tabela 7 mostra a relação entre Pessoas por FASFIL e o Índice de Gini que mede, nesse caso, o grau de desigualdade que existe na distribuição de indivíduos segundo a Renda domiciliar per capita. Quanto maior o valor, maior será a desigualdade de renda. Há uma correspon- 
Sylvio Bandeira de Mello e Silva, Barbara-Christine

Nentwig Silva, Maina Pirajá Silva

Tabela 5 - Unidades da Federação - Relação entre Pessoas por fundações privadas e associações sem fins lucrativos e PIB per capita - 2005

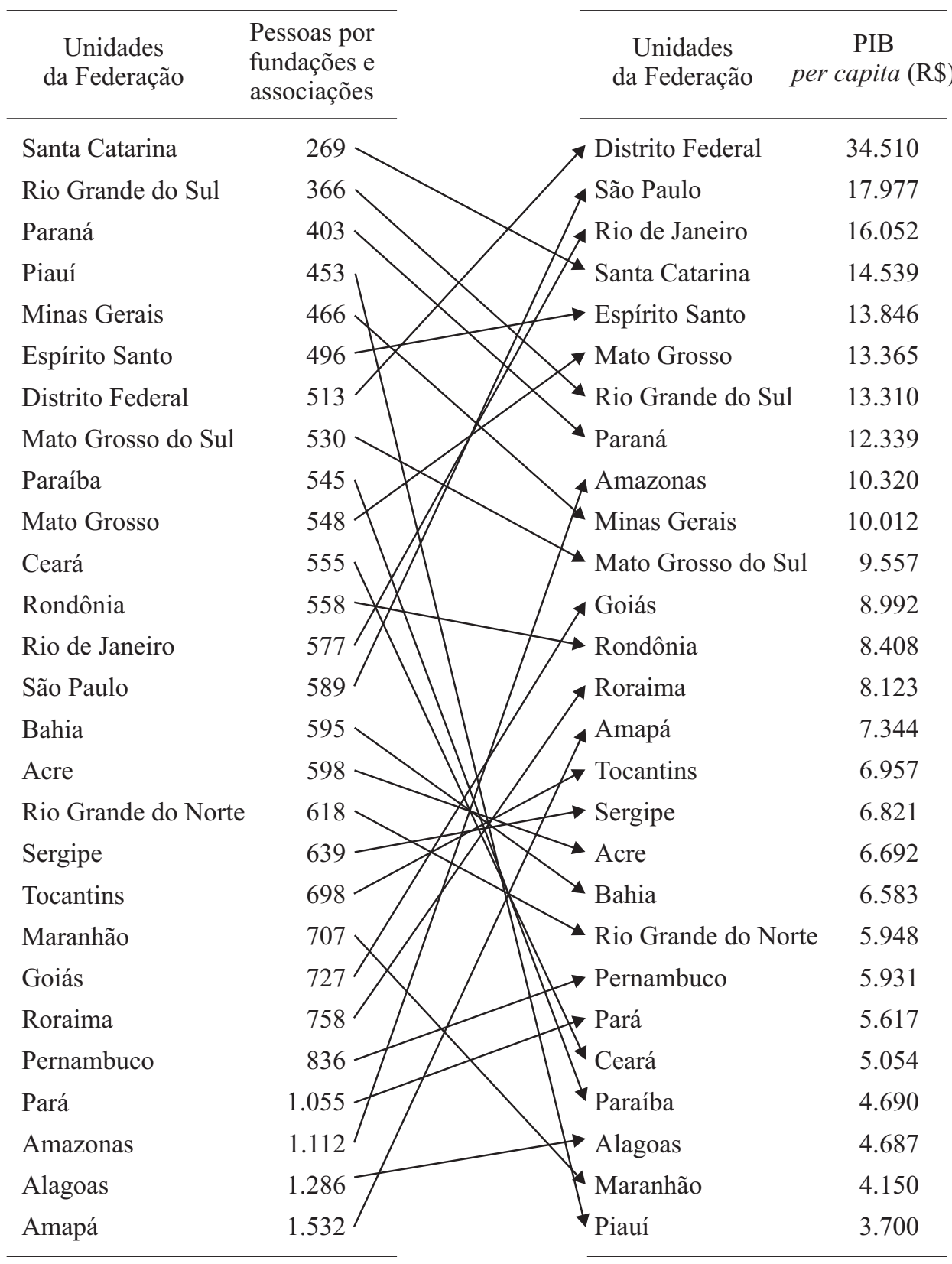

Fonte dos dados: IBGE, 2008; IBGE, 2002-2005. Elaboração: própria.

dência perfeita entre os dois indicadores em Santa Catarina. No total, oito estados mudam até três posições hierárquicas na comparação entre as duas variáveis. Merece destaque o Distrito Federal, com o mais alto Índice de Gini.
Já a Tabela 8 indica a relação entre as Pessoas por FASFIL e o IDH. Nessa relação estão 11 estados com mudança de posição em até três níveis. Destaca-se Santa Catarina, no $1^{\circ}$ lugar na análise pessoas por FASFIL, e, no $2^{\circ}$ lugar, nos índi- 
Tabela 6 - Unidades da Federação - Relação entre Pessoas por fundações privadas e associações sem fins lucrativos e o Índice de Williamson calculado com base em Microrregióes - 2005

\begin{tabular}{|c|c|c|c|}
\hline $\begin{array}{c}\text { Unidades } \\
\text { da Federação }\end{array}$ & $\begin{array}{l}\text { Pessoas por } \\
\text { fundações e } \\
\text { associações }\end{array}$ & $\begin{array}{c}\text { Unidades } \\
\text { da Federação }\end{array}$ & $\begin{array}{l}\text { Índice de } \\
\text { Williamson }\end{array}$ \\
\hline Santa Catarina & 269 & Amapá & 0,115 \\
\hline Rio Grande do Sul & 366 & Roraima & 0,149 \\
\hline Paraná & 403 & Mato Grosso do Sul & 0,201 \\
\hline Piauí & 453 & Acre & 0,213 \\
\hline Minas Gerais & 466 & Santa Catarina & 0,239 \\
\hline Espírito Santo & 496 & Paraná & 0,281 \\
\hline Distrito Federal & 513 & Distrito Federal* & - \\
\hline Mato Grosso do Sul & 530 & Tocantins & 0,300 \\
\hline Paraíba & 545 & São Paulo & 0,333 \\
\hline Mato Grosso & 548 & Rondônia & 0,344 \\
\hline Ceará & 555 & Rio de Janeiro & 0,378 \\
\hline Rondônia & 558 & Alagoas & 0,393 \\
\hline Rio de Janeiro & 577 & Rio Grande do Sul & 0,399 \\
\hline São Paulo & 589 & Espírito Santo & 0,402 \\
\hline Bahia & & Sergipe & 0,414 \\
\hline Acre & 598 & Rio Grande do Norte & 0,422 \\
\hline Rio Grande do Norte & 618 & Minas Gerais & 0,423 \\
\hline Sergipe & 639 & Goiás & 0,431 \\
\hline Tocantins & 698 & Pará & 0,440 \\
\hline Maranhão & 707 & Piauí & 0,453 \\
\hline Goiás & 727 & Ceará & 0,465 \\
\hline Roraima & $758 /$ & Paraíba & 0,489 \\
\hline Pernambuco & 836 & Amazonas & 0,546 \\
\hline Pará & 1.055 & Maranhão & 0,629 \\
\hline Amazonas & 1.112 & Mato Grosso & 0,672 \\
\hline Alagoas & $1.286 \%$ & Bahia & 0,677 \\
\hline Amapá & 1.532 & Pernambuco & 0,701 \\
\hline
\end{tabular}

* Como o Distrito Federal não é dividido em Microrregiões, não é possível calcular o Índice de Williamson.

Fonte dos dados: IBGE, 2008; IBGE, 2002-2005.

Elaboração: própria.

ces do IDH. O Distrito Federal, Santa Catarina e São Paulo ocupam os três primeiros lugares, respectivamente, no IDH. Os piores Índice de desenvolvimento humano estão no Nordeste.

Por outro lado, a relação entre Pessoas por
FASFIL e Taxas de mortalidade infantil apresenta 12 estados com variação de posição de até três níveis (Tabela 9). Os primeiros quatro lugares em baixos índices de Mortalidade infantil são ocupados pelos estados do Rio Grande do Sul, São Pau- 
Sylvio Bandeira de Mello e Silva, Barbara-Christine

Nentwig Silva, Maina Pirajá Silva

Tabela 7 - Unidades da Federação - Relação entre Pessoas por fundações privadas e associações sem fins lucrativos e o Índice de Gini - 2005

\begin{tabular}{|c|c|c|c|}
\hline $\begin{array}{c}\text { Unidades } \\
\text { da Federação }\end{array}$ & $\begin{array}{l}\text { Pessoas por } \\
\text { fundações e } \\
\text { associações }\end{array}$ & $\begin{array}{c}\text { Unidades } \\
\text { da Federação }\end{array}$ & $\begin{array}{l}\text { Índice de } \\
\text { Gini }\end{array}$ \\
\hline Santa Catarina & $269-$ & Santa Catarina & 0,461 \\
\hline Rio Grande do Sul & $366-$ & Amazonas & 0,512 \\
\hline Paraná & 403 & Pará & 0,515 \\
\hline Piauí & 453 & Rio Grande do Sul & 0,520 \\
\hline Minas Gerais & 466 & Maranhão & 0,521 \\
\hline Espírito Santo & 496 & Mato Grosso & 0,523 \\
\hline Distrito Federal & 513 & Amapá & 0,526 \\
\hline Mato Grosso do Sul & 530 & Minas Gerais & 0,528 \\
\hline Paraíba & 545 & São Paulo & 0,532 \\
\hline Mato Grosso & 548 & Mato Grosso do Sul & 0,533 \\
\hline Ceará & 555 & Tocantins & 0,535 \\
\hline Rondônia & 558 & Paraná & 0,539 \\
\hline Rio de Janeiro & 577 & Roraima & 0,544 \\
\hline São Paulo & 589 & Bahia & 0,553 \\
\hline Bahia & 595 & Sergipe & 0,554 \\
\hline Acre & 598 & Rio de Janeiro & 0,557 \\
\hline Rio Grande do Norte & 618 & Goiás & 0,557 \\
\hline Sergipe & 639 & Espírito Santo & 0,558 \\
\hline Tocantins & 698 & Alagoas & 0,566 \\
\hline Maranhão & $707 /$ & Rondônia & 0,567 \\
\hline Goiás & 727 & Ceará & 0,579 \\
\hline Roraima & 758 & Paraíba & 0,581 \\
\hline Pernambuco & 836 & Acre & 0,583 \\
\hline Pará & $1.055 /$ & Pernambuco & 0,587 \\
\hline Amazonas & 1.112 & Piauí & 0,591 \\
\hline Alagoas & $1.286 /$ & Rio Grande do Norte & 0,598 \\
\hline Amapá & $1.532 /$ & Distrito Federal & 0,608 \\
\hline
\end{tabular}

Fonte dos dados: IBGE, 2008; IPEA, 2005.

Elaboração: própria.

lo, Santa Catarina e o Distrito Federal. Merecem nova referência os estados de Piaú, Paraíba e Ceará, com relativamente boa posição no indicador Pessoas por FASFIL e péssima posição nas Taxas de mortalidade infantil. O contexto geral dos estados nordestinos continua em má situação.
A relação entre Pessoas por FASFIL e Esperança de vida ao nascer mostra 16 estados com mudanças em suas posições hierárquicas em até três níveis. O Distrito Federal, Santa Catarina e Rio Grande do Sul tomam as primeiras três posições. Novamente os estados nordestinos ocupam 
Tabela 8 - Unidades da Federação - Relação entre Pessoas por fundações privadas e associações sem fins lucrativos e Índice de desenvolvimento humano - 2005

\begin{tabular}{|c|c|c|c|}
\hline $\begin{array}{c}\text { Unidades } \\
\text { da Federação }\end{array}$ & $\begin{array}{l}\text { Pessoas por } \\
\text { fundações e } \\
\text { associações }\end{array}$ & $\begin{array}{c}\text { Unidades } \\
\text { da Federação }\end{array}$ & IDH \\
\hline Santa Catarina & 269 & Distrito Federal & 0,874 \\
\hline Rio Grande do Sul & 366 & Santa Catarina & 0,840 \\
\hline Paraná & 403 & São Paulo & 0,833 \\
\hline Piauí & 453 & Rio de Janeiro & 0,832 \\
\hline Minas Gerais & 466 & Rio Grande do Sul & 0,832 \\
\hline Espírito Santo & 496 & Paraná & 0,820 \\
\hline Distrito Federal & 513 & Espírito Santo & 0,802 \\
\hline Mato Grosso do Sul & 530 & Mato Grosso do Sul & 0,802 \\
\hline Paraíba & 545 & Goiás & 0,800 \\
\hline Mato Grosso & 548 & Minas Gerais & 0,800 \\
\hline Ceará & 555 & Mato Grosso & 0,796 \\
\hline Rondônia & 558 & Amapá & 0,780 \\
\hline Rio de Janeiro & 577 & Amazonas & 0,780 \\
\hline São Paulo & $589^{\prime}$ & Rondônia & 0,776 \\
\hline Bahia & 595 & Tocantins & 0,756 \\
\hline Acre & 598 & Pará & 0,755 \\
\hline Rio Grande do Norte & 618 & Acre & 0,751 \\
\hline Sergipe & 639 & Roraima & 0,750 \\
\hline Tocantins & 698 & Bahia & 0,742 \\
\hline Maranhão & 707 & Sergipe & 0,742 \\
\hline Goiás & 727 & Rio Grande do Norte & 0,738 \\
\hline Roraima & 758 & Ceará & 0,723 \\
\hline Pernambuco & 836 & Pernambuco & 0,718 \\
\hline Pará & $1.055^{\prime}$ & Paraíba & 0,718 \\
\hline Amazonas & $1.112 /$ & Piauí & 0,703 \\
\hline Alagoas & $1.286 y$ & Maranhão & 0,683 \\
\hline Amapá & $1.532 /$ & Alagoas & 0,677 \\
\hline
\end{tabular}

Fonte dos dados: IBGE, 2008; PNUD, 2005.

Elaboração: própria.

os últimos lugares (Tabela 10).

Na comparação entre Pessoas por FASFIL e Média de anos de estudos das pessoas de 20 a 24 anos, há 11 estados que mudaram suas posições em até três níveis. Mais uma vez, São Paulo, Distrito Federal e Santa Catarina tomam os três primeiros lugares na Média de anos de estudo e os estados nordestinos as últimas posições (Tabela 11).

Finalmente, foi efetuada uma análise estatística de correlação, usando-se o coeficiente de correlação produto-momento de Karl Pearson, entre Pessoas por FASFIL e as variáveis apresenta- 
Sylvio Bandeira de Mello e Silva, Barbara-Christine

Nentwig Silva, Maina Pirajá Silva

Tabela 9 - Unidades da Federação - Relação entre Pessoas por fundações privadas e associaçóes sem fins lucrativos e Mortalidade infantil - 2005

\begin{tabular}{|c|c|c|c|}
\hline $\begin{array}{c}\text { Unidades } \\
\text { da Federação }\end{array}$ & $\begin{array}{l}\text { Pessoas por } \\
\text { fundações e } \\
\text { associações }\end{array}$ & $\begin{array}{c}\text { Unidades } \\
\text { da Federação }\end{array}$ & $\begin{array}{l}\text { Mortalidade } \\
\text { infantil* }\end{array}$ \\
\hline Santa Catarina & $269-$ & Rio Grande do Sul & 14,3 \\
\hline Rio Grande do Sul & $366-$ & São Paulo & 16,5 \\
\hline Paraná & 403 & Santa Catarina & 17,2 \\
\hline Piauí & 453 & Distrito Federal & 17,8 \\
\hline Minas Gerais & 466 & Mato Grosso do Sul & 19,1 \\
\hline Espírito Santo & 496 & Paraná & 20,0 \\
\hline Distrito Federal & 513 & Roraima & 20,1 \\
\hline Mato Grosso do Sul & 530 & Espírito Santo & 20,1 \\
\hline Paraíba & 545 & Goiás & 20,7 \\
\hline Mato Grosso & 548 & Rio de Janeiro & 20,9 \\
\hline Ceará & 555 & Mato Grosso & 21,6 \\
\hline Rondônia & 558 & Minas Gerais & 21,8 \\
\hline Rio de Janeiro & 577 & Rondônia & 25,2 \\
\hline São Paulo & $589^{\prime}$ & Amapá & 25,4 \\
\hline Bahia & 595 & Pará & 25,9 \\
\hline Acre & 598 & Amazonas & 27,6 \\
\hline Rio Grande do Norte & 010 & Tocantins & 29,0 \\
\hline Sergipe & 639 & Piauí & 30,4 \\
\hline Tocantins & & Ceará & 32,0 \\
\hline Maranhão & 707 & Acre & 32,6 \\
\hline Goiás & 727 & Bahia & 35,6 \\
\hline Roraima & 758 & Sergipe & 36,2 \\
\hline Pernambuco & & Rio Grande do Norte & 37,5 \\
\hline Pará & 1.055 & Paraíba & 40,8 \\
\hline Amazonas & $1.112^{\prime}$ & Pernambuco & 41,2 \\
\hline Alagoas & 1.2867 & Maranhão & 42,1 \\
\hline Amapá & $1.532 /$ & Alagoas & 53,7 \\
\hline
\end{tabular}

* Taxas de mortalidade infantil por 1.000 nascidos vivos.

Fonte dos dados: IBGE, 2008; IBGE, 2005.

Elaboração: própria.

das (Tabela 12). Para tanto, inicialmente, a correla- ções, como foi comentado anteriormente. Assim, ção entre todos os estados com seus indicadores por exemplo, em 16 estados brasileiros, a posição de Pessoas por FASFIL e cada variável revelou-se hierárquica entre Pessoas por FASFIL e Esperança baixa. Em seguida, questionou-se se não haveria de vida ao nascer não mudou mais de que três uma correlação significativa entre os estados e as posições hierárquicas, como vimos anteriormenvariáveis cujas mudanças na hierarquia nas duas te. No outro extremo, na relação entre Pessoas/ colunas apresentadas não superassem três posi- FASFIL e Índice de Williamson, somente sete es- 
Tabela 10 - Unidades da Federação - Relação entre Pessoas por fundações privadas e associações sem fins lucrativos e Esperança de vida ao nascer - 2005

\begin{tabular}{|c|c|c|c|}
\hline $\begin{array}{l}\text { Unidades } \\
\text { da Federação }\end{array}$ & $\begin{array}{l}\text { Pessoas por } \\
\text { fundações e } \\
\text { associações }\end{array}$ & $\begin{array}{l}\text { Unidades } \\
\text { da Federação }\end{array}$ & $\begin{array}{l}\text { Esperança } \\
\text { de vida } \\
\text { ao nascer }\end{array}$ \\
\hline Santa Catarina & 269 & Distrito Federal & 74,9 \\
\hline Rio Grande do Sul & 366 & $\rightarrow$ Santa Catarina & 74,8 \\
\hline Paraná & 403 & Rio Grande do Sul & 74,5 \\
\hline Piauí & 453 & Minas Gerais & 74,1 \\
\hline Minas Gerais & & São Paulo & 73,7 \\
\hline Espírito Santo & & Paraná & 73,5 \\
\hline Distrito Federal & 513 & Mato Grosso do Sul & 73,2 \\
\hline Mato Grosso do Sul & 530 & Espírito Santo & 73,1 \\
\hline Paraíba & 545 & Goiás & 72,8 \\
\hline Mato Grosso & & Mato Grosso & 72,6 \\
\hline Ceará & 555 & Rio de Janeiro & 72,4 \\
\hline Rondônia & 558 & Bahia & 71,4 \\
\hline Rio de Janeiro & & Pará & 71,4 \\
\hline São Paulo & 589 & Amazonas & 71,0 \\
\hline Bahia & 595 & Acre & 70,8 \\
\hline Acre & 598 & Tocantins & 70,7 \\
\hline Rio Grande do Norte & & Rondônia & 70,6 \\
\hline Sergipe & 639 & $\rightarrow$ Sergipe & 70,3 \\
\hline Tocantins & 698 & Amapá & 69,8 \\
\hline Maranhão & & Rio Grande do Norte & 69,8 \\
\hline Goiás & 727 & 'Ceará & 69,6 \\
\hline Roraima & 758 & Roraima & 69,3 \\
\hline Pernambuco & & Paraíba & 68,3 \\
\hline Pará & $1.055^{\prime}$ & Piauí & 68,2 \\
\hline Amazonas & $1.112 /$ & Pernambuco & 67,5 \\
\hline Alagoas & $1.286-$ & Maranhão & 66,8 \\
\hline Amapá & $1.532 /$ & Alagoas & 66,0 \\
\hline
\end{tabular}

Fonte dos dados: IBGE, 2008; IBGE, 2005.

Elaboração: própria.

tados não mudaram, segundo a nossa definição, mais de três posições hierárquicas. A análise, neste caso, indica correlações altas para todas as variáveis, à exceção do PIB per capita, mas cujo valor ainda pode ser considerado significativo. (Gerardi; Silva, 1981, p.98-104, 160)
O Mapa 2 ressalta, como síntese, os estados com altas correlações entre Pessoas/FASFIL e as variáveis apresentadas, onde se destacam Santa Catarina, Sergipe e Pernambuco, que apresentam um total de seis altas correlações das sete análises de correlação efetuadas. Como se percebe, o resul- 
Sylvio Bandeira de Mello e Silva, Barbara-Christine

Nentwig Silva, Maina Pirajá Silva

Tabela 11 - Unidades da Federação - Relação entre Pessoas por fundações privadas e associações sem fins lucrativos e Média de anos de estudo das pessoas de 20 a 24 anos -2005

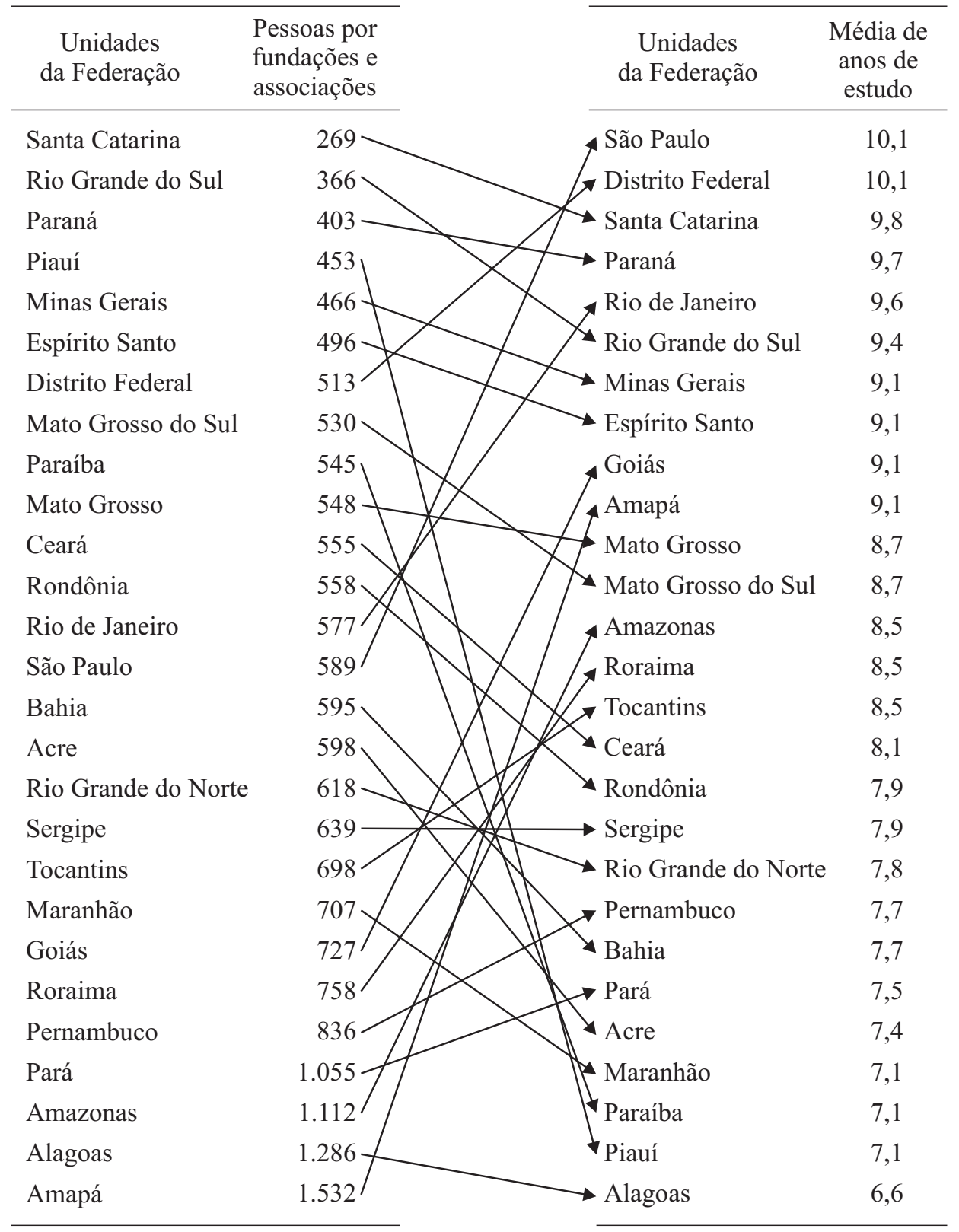

Fonte dos dados: IBGE, 2008; IBGE, 2005.

Elaboração: própria.

tado integra um estado com melhores indicadores, Santa Catarina, e dois estados com indicadores menos favoráveis, Sergipe e Pernambuco, o que mereceria outras análises. Os estados com nenhu- do país. 
Tabela 12 - Correlação entre Pessoas por FASFIL e variáveis - 2005

\begin{tabular}{l|c|c}
\hline Pessoas por FASFIL e variáveis & $\begin{array}{c}\text { Coeficiente de correlação } \\
\text { (r) }\end{array}$ & $\begin{array}{c}\text { Estados com mudanças até 3 } \\
\text { posiçóes hierárquicas }\end{array}$ \\
\hline Índice de Williamson & 0,958809324 & 7 \\
\hline Mortalidade infantil & 0,956510763 & 12 \\
\hline Índice de Gini & 0,940882621 & 11 \\
\hline IDH & $-0,942710986$ & 16 \\
\hline Esperança de vida ao nascer & $-0,931828471$ & 10 \\
\hline Média de anos de estudo (20 a 24 anos) & $-0,923131509$ & 11 \\
\hline PIB per capita & $-0,759775592$ & \\
\hline
\end{tabular}

Fonte dos dados: IBGE, 2008; IBGE, 2005; IBGE, 2002-2005; IPEA, 2005; PNUD, 2005.

Elaboração: própria.

Mapa 2 - Brasil - Unidades da Federação - Número de altas correlações

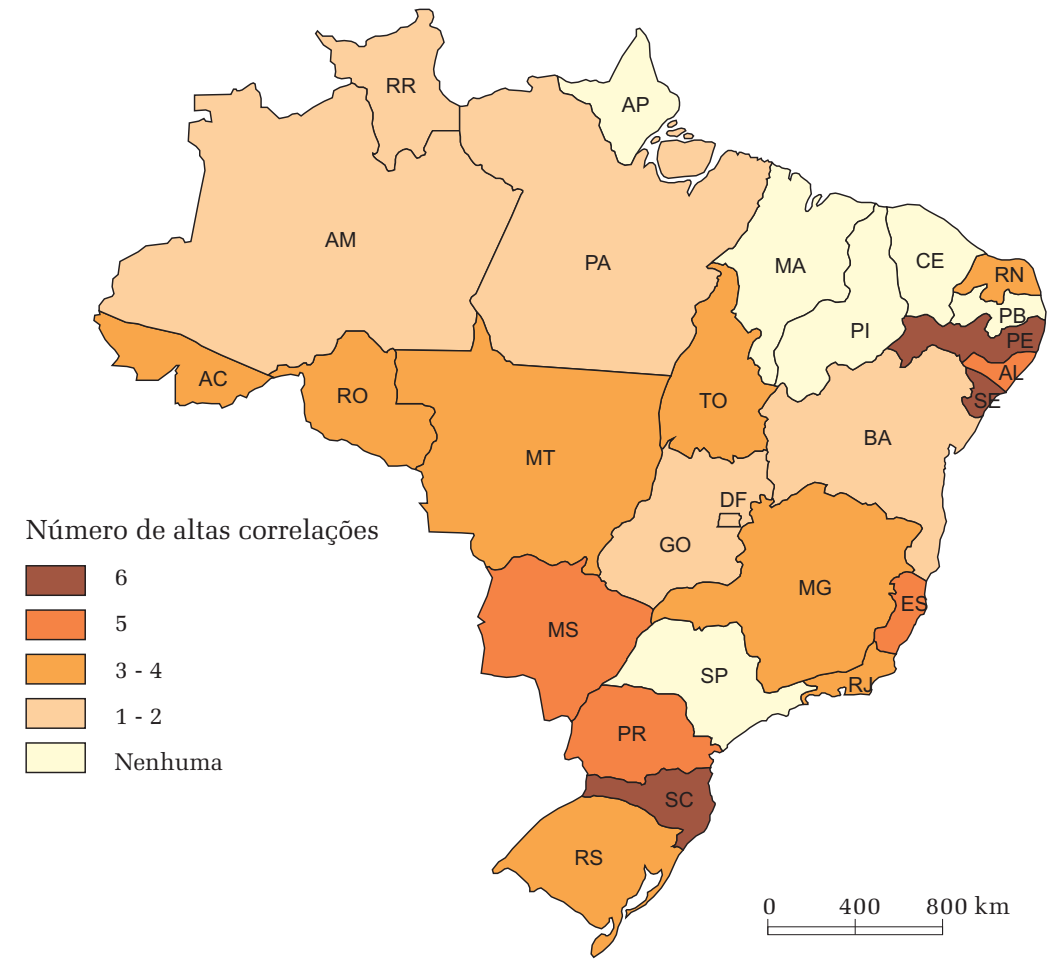

Fonte dos dados: IBGE, 2008; IBGE, 2005; IBGE, 2002-2005;

IPEA, 2005; PNUD, 2005.

Elaboração: própria.

\section{CONCLUSÃO}

A abordagem da organização social, através do capital social, permite ressaltar a relevância da temática em sua conexão com a análise dos diversificados indicadores socioeconômicos brasileiros.

Assim, a análise da organização social no Brasil e nos estados brasileiros, medida através das fundações privadas e associações sem fins lucrativos, seguindo uma das mais importantes contribuições de Putnam (1996), em sua relação com os desequilíbrios e desigualdades, revela importantes questões que merecem ser destacadas, indicando futuros desdobramentos.

A primeira delas é a de que há uma grande variedade na distribuição dos indicadores de or- 
ganização social que precisaria ser explicada através de uma geografia histórica fortemente apoiada na análise econômica, sociocultural e política. Os estados do Sul, especialmente Santa Catarina e Rio Grande do Sul, estão em melhor situação com relação ao capital social que os do Nordeste e do Norte do país. Os estados do Sudeste ocupam uma posição intermediária.

Já nas comparações decorrentes das relações entre Pessoas por fundações e associações sem fins lucrativos com diversos indicadores (PIB percapita, Índice de Williamson, Mortalidade infantil, Índice de Gini, IDH, Esperança de vida ao nascer e Média de anos de estudo - 20 a 24 anos), observa-se uma expressiva diversidade de situações.

Com efeito, as análises demonstraram que 15 estados apresentam boas relações com pelo menos três variáveis das sete que foram pesquisadas. O destaque é para o estado de Santa Catarina, o $1^{\circ}$ em Pessoas por FASFIL e em seis variáveis, PIB per capita, Índice de Gini, IDH, Esperança de vida ao nascer, Mortalidade infantil e Média de anos de estudo, ocupa a $4^{\mathrm{a}}$, a $1^{\mathrm{a}}$ e duas vezes a $2^{\mathrm{a}}$ e a $3^{\mathrm{a}}$ posição hierárquica, respectivamente. É um dos estados que apresenta o maior número de altas correlações entre Pessoas por FASFIL e variáveis. Por outro lado, os estados de Sergipe e Pernambuco, que se destacam igualmente com seis altas correlações das sete variáveis escolhidas, apresentam posições muito baixas em todos os indicadores. Isso significa dizer que Santa Catarina integra, em posições elevadas, os indicadores escolhidos, enquanto que Sergipe e Pernambuco integram, em baixas posições, os mesmos indicadores.

Assim, com base neste estudo, não é possível afirmar que há uma relação determinística entre níveis elevados de organização social e melhores indicadores socioeconômicos em todo o Brasil, mas há sinais positivos que apontam para essa direção.

Existe, portanto, um potencial de análises e reflexões que merece ser explorado, tentando aprofundar o conhecimento sobre a relação entre organização social e desenvolvimento econômico- social no Brasil. Isso certamente exigirá um estudo de muito maior fôlego, sendo preciso lembrar que a pesquisa conduzida por Putnam com sua equipe, na Itália, demorou 20 anos para ser concluída.

Um bom exemplo já analisado em nosso país, na escala estadual, é justamente o de Santa Catarina, com os seus Fóruns de Desenvolvimento Regional Integrado (Birkner, 2006). O autor conclui que eles "geraram muitos dos efeitos esperados, embora insuficientes", sendo um "exemplo viável àqueles que, por disposição política ou interesse científico, procuram identificar processos políticos capazes de gerar ambientes de sinergia e construção de capital social” (Birkner, 2006, p.169). A situação de Santa Catarina foi também comparada com a da Bahia por Silva, Silva e Coelho (2008), quando foi possível revelar a pertinência da relação entre capital social e níveis de desenvolvimento estadual. O exemplo do Rio Grande do Sul, outro estado bem colocado nas análises anteriores, foi estudado e avaliado positivamente por Monastério (2001) e por diversos trabalhos de Bandeira (2004), dentre outros.

Na Bahia, duas pesquisas valorizam a escala municipal (Valente) associada à escala microrregional (região do sisal). São os de Nascimento (2003) e Silva e Silva ([2003] 2006). Esses estudos destacam a importância da Associação de Desenvolvimento Sustentável e Solidário da Região Sisaleira (APAEB), fundada e dirigida por pequenos produtores de sisal.

A expectativaé de que este trabalho sobre todo o Brasil, que complementa os estudos isolados, já realizados nas escalas estaduais, microrregionais e municipais, possa motivar outras análises integradas para o aprofundamento da relação entre organização social e desenvolvimento socioeconômico na escala nacional e em outras escalas e contextos da realidade brasileira. Como diz Putnam (1996, p.194), “criar capital social não será fácil, mas é fundamental para fazer a democracia funcionar".

(Recebido para publicação em junho de 2009) (Aceito em setembro de 2009) 


\section{REFERÊNCIAS}

ARAÚJO, M. C. D’. Capital social. Rio de Janeiro: Jorge Zahar , 2003.

BANDEIRA, P. S. Articulação de atores sociais, capita social e desenvolvimento regional: o caso dos Conselhos de Desenvolvimento do Rio Grande do Sul. In: SEI. Superintendência de Estudos Econômicos e Sociais da Bahia (Org.) Desigualdades regionais. Salvador: 2004. v.67. p.219-250.

BARROSO, I. C.; ROMERO, G. G. Las redes de colaboración como base del desarrollo territorial. Scripta Nova, Barcelona, v.13, n. 289, p.1-23, 2009.

BASCO, M. C.; LAXALDE, M. del P. F. Economía solidaria y capital social. Buenos Aires: Paidós, 2005.

BECKER, D. Capital social: uma nova derivação da economia de mercado? In: CORREA, S. M. de S. (Org.) Capita social e desenvolvimento regional. Santa Cruz do Sul,RS EDUNISC, 2003. p.85-121.

BIRKNER, W. M. K. Capital social em Santa Catarina: o caso dos Fóruns de Desenvolvimento Regional. Blumenau: EDIFURB, 2006.

BOURDIEU, P. O capital social - notas provisórias. In: NOGUEIRA, M. A.; CATANI, A. (Org.) Pierre Bourdieu escritos de educação. Petrópolis: Vozes, [1980] 1998

COLEMAN, J. S. Social capital in the creation of human capital. American Journal of Sociology, Chicago, v.94, p.95120, 1988. (Supplement)

Foundations of social theory. Cambridge: Harvard University Press, 1990.

CORREA, S. M. de S. (Org.) Capital social e desenvolvimento regional. Santa Cruz do Sul,RS: EDUNISC, 2003.

DINIZ, C. C. Desenvolvimento poligonal no Brasil: nem desconcentração nem continua polarização. Nova Economia, Belo Horizonte, v.3, n.1, p.35-64, 1993.

A nova geografia econômica do Brasil condicionantes e implicações. In: VELOSO, J. P. R. (Org.) Brasil século XX. Rio de Janeiro: José Olímpio, 2000.

GERARDI, L. H. de O.; SILVA, B. C. N. Quantificação em geografia. São Paulo: Difel, 1981.

HARVEY, D. Spaces of hope. Berkeley ; Los Angeles: University of California Press, 2000.

Spaces of capital. Towards a critical geography. New York: Routledge, 2001.

Do administrativismo ao empreendedorismo: a transformação da governança urbana no capitalismo tardio. In: . A produção capitalista do espaço. São Paulo: Annablume, [1989] 2005. p.165-190.

IBGE. As fundações privadas e associações sem fins lucrativos no Brasil 2005. Rio de Janeiro: 2008.

Contas regionais. Produto Interno Bruto dos $\mathrm{Mu}$ nicípios 2002-2005. Disponível em: http://www.ibge.gov.br/ home/estatistica/economia/pibmunicipios/2005/ default.shtm>. Acesso em: 09 set. 2008.

Síntese de indicadores sociais 2005. Disponível em: <http://www.ibge.gov.br/ home/estatistica/populacao/ c on d i c a o d e vida/indica d o r e s m i n i m o s/ sinteseindicsociais2006/default.shtm>. Acesso em: 09 set. 2008

IPEA. Índice de Gini 2005. Disponível em: <http:// w w w. i p e a d a t a.g o v. b r / i p e a w e b.d l l ipeadata?SessionID $=756025009 \&$ Tick $=1221050977227$ \&VAR FUNCA =SubmeterFormulario\%28\%27frmMain
\%27\%2C\%27Series\%3FSessionID\%3D756025009\%26 Text\%3DGINI\%26Tick\%3D1221050977227\%27\%29\% 3B\&Mod $=\mathrm{S}>$. Acesso em: 09 set. 2008.

MONASTÉRIO, L. M. O capital social e crescimento econômico no Rio Grande do Sul. Curitiba: Ed. UFPR, 2001.

NASCIMENTO, H. M. do. Conviver o sertão. Origem e evolução do capital social em Valente/BA. São Paulo: Annablume: Fapesp; Valente: Apaeb, 2003.

PNUD. Índice de Desenvolvimento Humano 2005. Disponível em: <http://www.pnud.org.br/ pobreza desigualdade/ reportagens/index.php?id01 $=3039 \& l a y=$ pde $>$. Acesso em: 09 jun. 2009.

PUTNAM, R. D. Comunidade e democracia: a experiência da Itália moderna. Rio de Janeiro: FGV, [1993] 1996.

SANTOS, M.; SILVEIRA, M. L. O Brasil. Território e sociedade no início do século XXI. Rio de Janeiro: Record, 2001.

SILVA, S. B. de M. e; SILVA, B. C. N. Reinventando o território: tradição e mudança na região do sisal - Bahia. In: $\quad$; Estudos sobre globalização, território e Bahia. Salvador: UFBA; Mestrado em Geografia, [2003]. 2006.

; COELHO, A. S. Desequilíbrios e desi-

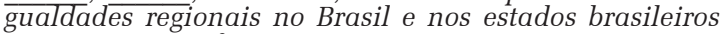
João Pessoa: Grafset, 2008.

SOTO, W. H. G. A dialética do desenvolvimento regional: capital social, democracia, redes empresariais e dinâmica territorial. In: BECKER, D. F; WITTMANN, M. L. Desenvolvimento regional: abordagens interdisciplinares. Santa Cruz do Sul; EDUNISC, 2003. p.383-395

WILLIAMSON, J.G. Desigualdade regional e o processo de desenvolvimento nacional: descrição dos padrões. In: SCHWARTZMAN, J. (Org.) Economia regional. Belo Horizonte: Cedeplar, [1965]. 1977. p.53-116. (Textos escolhidos).

WITTMANN, M. L.; RAMOS, M. P. (Org.) Desenvolvimento regional: capital social, redes e planejamento. Santa Cruz do Sul, RS: EDUNISC, 2004.

WOOLCOCK, M. Social capital and economic development: toward a theoretical synthesis and policy framework. Theory and Society, Dordrech, NL, v. 27, p.151208, 1998. 


\section{SOCIAL ORGANIZATION AND SOCIAL AND ECONOMIC INDICATORS IN BRAZIL: an exploratory study}

\author{
Sylvio Bandeira de Mello e Silva, Barbara- \\ Christine Nentwig Silva, Maina Pirajá Silva
}

The purpose of this work is to analyze the spatial distribution of social capital in Brazil and correlate these results with several indicators of social economic development. Information gathered from IBGE on Private and Non-Profitable Associations that play an important role in society were used. The indicators of social-economic development are the following: Williamson index of regional unbalance, GDP per capita, Human Development Index (HDI), Gini index, rate of infant mortality, life expectancy at birth and school year mean (20-24 years). We used a methodology of statistical and cartographical analysis in different scales of Brazil's territory. The results showed a great variety of situations. However, it is possible to assert that there is a significant correlation between better indicators of social-economic development in states with a greater balance between its number of inhabitants and the presence of social organizations. Therefore, the results are relevant for understanding Brazil's geographic variety.

Keywords: social organization, social capital, socioeconomic indicators, development, Brazil.

\section{ORGANISATION SOCIALE ET INDICATEURS SOCIO-ECONOMIQUES AU BRESIL: une etude exploratoire}

\author{
Sylvio Bandeira de Mello e Silva, Barbara- \\ Christine Nentwig Silva, Maina Pirajá Silva
}

Lobjectif de cette étude est d'analyser la distribution spatiale des organisations sociales au Brésil et de faire la corrélation de ces résultats avec des données socio-économiques. Les informations fournies par l'IBGE concernant les fondations privées et les associations sans buts lucratifs ont servi d'indicateurs pour les organisations sociales qui jouent un rôle dans la société. Les indicateurs socioéconomiques sont les suivants: l'indice des déséquilibres régionaux de Williamson, le PIB par habitant, l'indice de développement humain (IDH), l'indice de Gini, le taux de mortalité infantile, l'espérance de vie à la naissance et la durée moyenne d'études (de 20 à 24 ans). Après avoir utilisé une méthodologie d'analyse statistique et cartographique, à différentes échelles du territoire national, les résultats témoignent d'une variété de situations. Il est cependant possible d'affirmer qu'il y a une corrélation significative avec des indicateurs économiques et sociaux meilleurs pour les états où le rapport entre personnes et organisations sociales est meilleur aussi. Ces résultats ont donc une importance fondamentale pourcomprendrela diversitégéographiquebrésilienne.

MотS-CLÉs: organisation sociale, capital social, indices sociaux économiques, développement, Brésil.

Sylvio Bandeira de Mello e Silva - Doutor em Geografia pela Universidade de Toulouse/França. Professor e Coordenador do Programa de Pós-Graduação em Planejamento Territorial e Desenvolvimento Social da Universidade Católica do Salvador/UCSAL e do Mestrado em Geografia da Universidade Federal da Bahia/ UFBA. Integra, como pesquisador/CNPq, o Núcleo de Pesquisa GLOCAL, desenvolvendo pesquisas na área de Planejamento Urbano/Regional e Geografia Urbana e Regional. Publicações recentes: Desequilíbrios e desigualdades regionais no Brasil e nos estados brasileiros. Livro em colaboração com B. C. N. Silva e A. S. Coelho. João Pessoa: Ed. Grafset, 2008. Mudanças na ocupação econômica no Litoral Sul da Bahia: os exemplos de Belmonte e Canavieieras-BA. Artigo em colaboração com D. M. C. Nascimento e J. M. L. Dominguez. Revista Desenbahia, v.5, 2009.

Barbara-Christine Nentwig Silva - Doutora em Geografia pela Universidade de Freiburg/Alemanha. Professora do Programa de Pós-Graduação em Planejamento Territorial e Desenvolvimento Social da Universidade Católica do Salvador/UCSAL e do Mestrado em Geografia da Universidade Federal da Bahia/UFBA. Integra, como pesquisadora/CNPq, o Núcleo de Pesquisa GLOCAL, desenvolvendo pesquisas na área de Cartografia, Análise de Dados, Planejamento Urbano/Regional e Geografia Urbana e Regional. Publicações recentes: Desequilíbrios e desigualdades regionais no Brasil e nos estados brasileiros. Livro em colaboração com S. B. M. Silva e A. S. Coelho (João Pessoa: Ed. Grafset, 2008). Elaboração do mapa de zonas de desenvolvimento da Região Metropolitana de Salvador através de modelagem cartográfica. Artigo em colaboração com S. S. de Carvalho. Geografia, Rio Claro, v. 34, 2009.

Maina Pirajá Silva - Licenciada e Bacharelanda em Geografia pela Universidade Federal da Bahia. Integra o Núcleo de Pesquisa GLOCAL/CNPq, desenvolvendo pesquisas na área de Cartografia, Geoprocessamento e Geografia Urbana e Regional. Publicações mais recentes: Rede urbana e educação em Roraima. Artigo em colaboração com S. B. M. Silva e B. C. N. Silva. RDE. Revista de Desenvolvimento Econômico, v. 18, p. 5-14, 2008. Estruturas etárias da população do Brasil e dos estados brasileiros. Em colaboração com: S. B. M. Silva; B. C. N. Silva e A. S. Coelho. RDE. Revista de Desenvolvimento Econômico, v. 9, 2007. 
